The University of Southern Mississippi

The Aquila Digital Community

Faculty Publications

7-1-2007

\title{
Measurement of Population Dynamics In Stimulated Raman Adiabatic Passage
}

\author{
Alina Gearba \\ University of Southern Mississippi, Alina.Gearba@usm.edu \\ H. A. Camp \\ Kansas State University \\ M. L. Trachy \\ Kansas State University \\ G. Veshapidze \\ Kansas State University \\ M.H. Shah \\ Kansas State University
}

See next page for additional authors

Follow this and additional works at: https://aquila.usm.edu/fac_pubs

Part of the Physics Commons

\section{Recommended Citation}

Gearba, A., Camp, H., Trachy, M., Veshapidze, G., Shah, M., Jang, H., DePaola, B. (2007). Measurement of Population Dynamics In Stimulated Raman Adiabatic Passage. Physical Review A, 76(1).

Available at: https://aquila.usm.edu/fac_pubs/1977

This Article is brought to you for free and open access by The Aquila Digital Community. It has been accepted for inclusion in Faculty Publications by an authorized administrator of The Aquila Digital Community. For more information, please contact Joshua.Cromwell@usm.edu. 
Authors

Alina Gearba, H. A. Camp, M. L. Trachy, G. Veshapidze, M.H. Shah, H.U. Jang, and B.D. DePaola 


\title{
Measurement of population dynamics in stimulated Raman adiabatic passage
}

\author{
M. A. Gearba, ${ }^{1,2}$ H. A. Camp, ${ }^{2, *}$ M. L. Trachy, ${ }^{2}$ G. Veshapidze, ${ }^{2}$ M. H. Shah,${ }^{2, \dagger}$ H. U. Jang, ${ }^{2}$ and B. D. DePaola ${ }^{2}$ \\ ${ }^{1}$ Department of Physics and Astronomy, University of Southern Mississippi, Hattiesburg, Mississippi 39406, USA \\ ${ }^{2}$ J. R. Macdonald Laboratory, Department of Physics, Kansas State University, Manhattan, Kansas 66506-2601, USA
}

(Received 10 May 2007; published 17 July 2007)

\begin{abstract}
The temporal evolution of populations has been directly measured for a three-level ladder system undergoing coherent excitation by stimulated Raman adiabatic passage (STIRAP). The measurement technique makes use of charge transfer as diagnostic. The method is model independent and has a temporal resolution of a few nanoseconds. The temporal evolution is measured for several values of the delay between the pump and Stokes laser pulses that are part of the STIRAP excitation scheme. The corresponding quantum Liouville equations are solved and the results of the calculations are compared with experiment.
\end{abstract}

DOI: 10.1103/PhysRevA.76.013406

PACS number(s): 32.80.Qk, 32.80.Pj, 34.70.+e, 42.50.Hz

\section{INTRODUCTION}

During the past decade a great deal of work, both theoretical [1-3] and experimental [4-6], has been invested in exploring efficient coherent excitation techniques in order to control population transfer between specified atomic and molecular states. The efficient preparation of a system in a desired state is important for advances in quantum information [7]; population transfer using coherent excitation has already been successfully employed to transfer population between metastable levels in cold, trapped calcium ions [8] for qubit readout purposes. Other areas that can benefit from efficient control of populations include atom optics [9], laser-assisted chemistry [10], creation of quantum degenerate molecules $[11,12]$, and solid-state physics [13]. Efficient coherent population transfer of ground-state atoms into Rydberg states has also recently been demonstrated $[6,14]$. In a recent intriguing experiment, it was shown that high-order harmonic generation $(\mathrm{HHG})$ could be readily generated from $\mathrm{Rb}(4 d)$ [15]. One might infer from this that efficient production of $\mathrm{Rb}(4 d)$ could therefore be instrumental in large scale HHG.

Stimulated Raman adiabatic passage (STIRAP) [16] is a robust technique used to coherently transfer population from an initial state to a desired final state, via an intermediate state, with little or no accumulation of population in the intermediate state. Note that if the intermediate state has a very short lifetime or can undergo undesirable chemical reactions, then leaving that state unpopulated can be as important as getting large transfer efficiencies to the target state. The most efficient population transfer is predicted to occur for the socalled counter-intuitive order, in which the optical pulse connecting the intermediate and final states precedes the optical pulse connecting the initial and the intermediate states. Depending on the details of the system, up to $100 \%$ of the population can be transferred from the ground state to the

\footnotetext{
*Present address: Institute for Defense Analyses, Science and Technology Division, 4850 Mark Center Drive, Alexandria, VA 22311-1882.

${ }^{\dagger}$ Present address: FOCUS Center and Michigan Center for Theoretical Physics, Department of Physics, University of Michigan, Ann Arbor, MI 48109.
}

desired final state. Furthermore, unlike schemes relying on Rabi flopping, the population transfer efficiency of STIRAP should not be sensitive to laser intensity or pulse length.

In most experiments the efficiency of the STIRAP process has been determined by measuring the fluorescence emitted by the desired final state $[16,17]$. Furthermore, in most STIRAP experiments, the absolute scale of transfer efficiency was fixed through comparison with theoretical models. Until now, no experiments have measured population evolution in all levels involved in the coherent excitation, nor have they measured the dynamics of population transfer. A measurement of the temporal evolution of the population during coherent excitation would provide an important test of the theoretical models that are being used to predict the success of procedures relying on STIRAP for efficient preparation of atomic and molecular systems. In the work presented here, we address this issue by employing a measurement technique, known as magneto-optical trap recoil ion momentum spectroscopy (MOTRIMS), that makes use of an ion beam as a probe of population dynamics. We apply MOTRIMS to a three-level ladder system in ${ }^{87} \mathrm{Rb}$ that is coherently excited using STIRAP. In our experiments the populations of all states involved in the STIRAP process are measured, with a temporal resolution of a few nanoseconds. That is, the populations of all states are measured as a function of time during the coherent excitation process. The measured fractional populations are measured on an absolute scale; the measurements do not rely on any theoretical model for normalization. The population evolution is measured as a function of the temporal delay between the two excitation laser pulses. Experimental results are compared with predictions of theory $[18,19]$.

The paper is organized as follows: A summary of the theoretical model used to characterize STIRAP is given in Sec. II. Section III contains a brief description of the MOTRIMS apparatus, along with the methodology used to measure the population dynamics during coherent excitation. Section IV presents the experimental data and comparison with predictions of theory. Finally, Sec. V contains a summary of the work presented in the paper as well as plans for future experiments. 

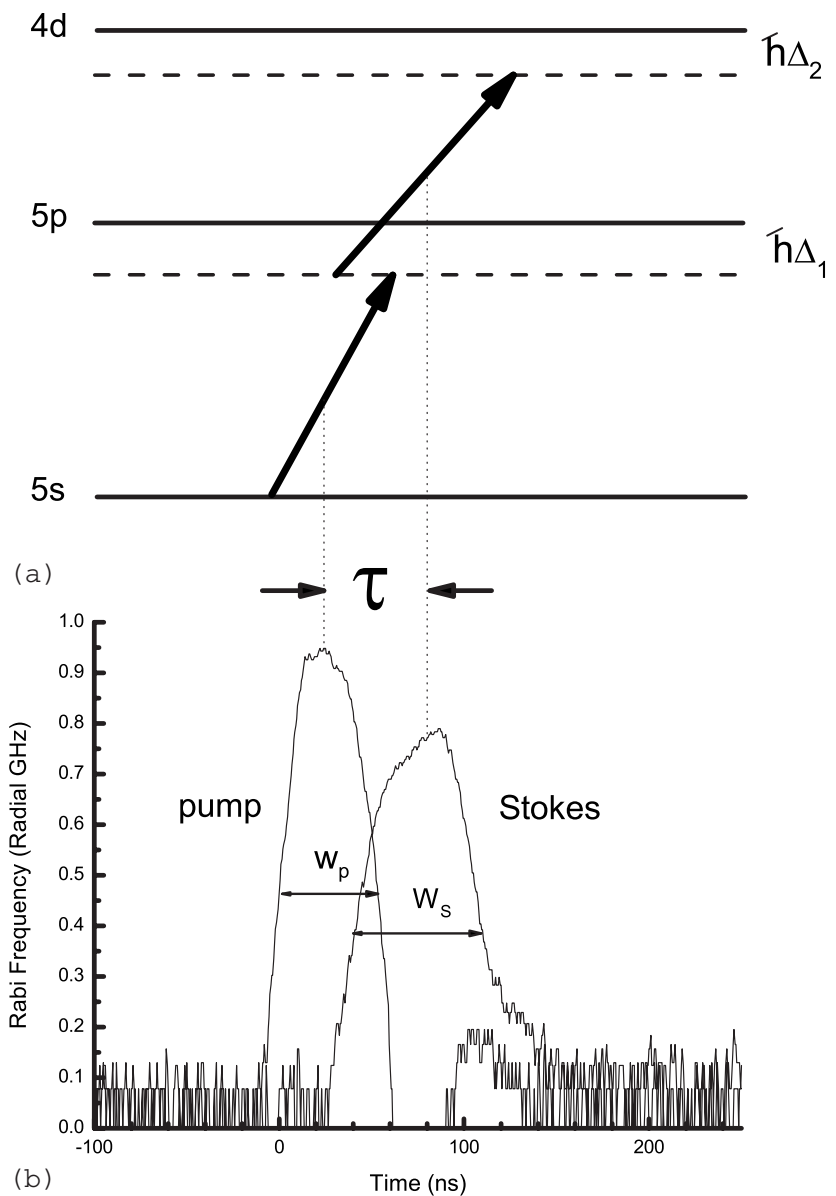

FIG. 1. (a) Partial energy level diagram showing the pump and Stokes transitions. (b) Laser pulse sequence. In this case, the pump laser arrives before the Stokes laser in the so-called "intuitive" order, for which $\tau$ is defined to be a positive quantity.

\section{THEORY}

Studies of coherent population transfer in atomic or molecular systems can be found extensively in the literature $[1,2]$. Here, only a brief summary of the theory will be given, as applied to a three-level ladder system, coherently excited via STIRAP. Consider the three-level $\mathrm{Rb}$ ladder system shown in Fig. 1(a). The levels of interest are ${ }^{87} \mathrm{Rb}\left(5 s_{1 / 2}\right), F$ $=2 ;{ }^{87} \mathrm{Rb}\left(5 p_{3 / 2}\right), F=3$; and ${ }^{87} \mathrm{Rb}\left(4 d_{5 / 2}\right)$ [20]. For convenience, we drop reference to the hyperfine levels in Fig. 1 and throughout the remainder of this paper. Light from a $780 \mathrm{~nm}$ laser, the so-called pump laser, is tuned to the red of the $5 s_{1 / 2} \rightarrow 5 p_{3 / 2}$ transition by an energy $\hbar \Delta_{1}$, while a $1529 \mathrm{~nm}$ laser, the so-called Stokes laser, is tuned to the blue of the $5 p_{3 / 2} \rightarrow 4 d_{5 / 2}$ transition by an energy $\hbar \Delta_{2}$. Then, $\hbar \Delta_{1}$ and $\hbar \Delta_{1}+\hbar \Delta_{2}$ are the detunings from one- and two-photon resonance, respectively. The Rabi frequencies of the pump and Stokes lasers are $\Omega_{p}$ and $\Omega_{s}$, respectively. Figure 1 shows how the relative timing between the two laser pulses, $\tau$, is defined. Efficient transfer is predicted to occur when the pump and Stokes lasers are pulsed in the counterintuitive order, that is, when the Stokes laser pulse precedes the pump laser pulse, for which case $\tau$ is defined to be negative. The process of stimulated Raman adiabatic passage can be described [16] as follows: The Stokes laser first dresses the system. The pump laser then couples the initial state with the dressed system, allowing population to move from the initial ground state to one of the dressed system's eigenstates. While the pump laser is still on, light from the Stokes laser is gradually reduced, thus adiabatically allowing the system to evolve back into the undressed form, and "stranding" the population in the uppermost state of the undressed system. If the system is allowed to evolve adiabatically, by which is meant the laser intensities vary slowly compared to the corresponding Rabi periods, then if spontaneous emission is totally neglected, $100 \%$ of the population will remain in the $\mathrm{Rb}\left(4 d_{5 / 2}\right)$ state. Even with the finite lifetimes of $\sim 90 \mathrm{~ns}$ for the $4 d_{5 / 2}$ state and $\sim 27 \mathrm{~ns}$ for the $5 p_{3 / 2}$ state, a peak population of nearly $90 \%$ is predicted for the $4 d_{5 / 2}$ state.

A convenient method for describing the evolution of the system during coherent excitation is through the use of density matrices. Using the rotating-wave approximation, the Hamiltonian describing the coupling of the three states by the two coherent radiation fields can be written $[1-4,16]$ as

$$
H=\frac{\hbar}{2}\left(\begin{array}{ccc}
0 & \Omega_{p} & 0 \\
\Omega_{p}^{*} & 2 \Delta_{1} & \Omega_{s} \\
0 & \Omega_{s}^{*} & 2\left(\Delta_{1}+\Delta_{2}\right)
\end{array}\right) .
$$

The quantum Liouville equation, which includes relaxation terms to account for losses, and more importantly loss of coherence due to spontaneous emission, can then be written $[1]$ as

$$
\iota \hbar \dot{\rho}_{i j}=[H, \rho]-i \hbar[\Gamma \rho]_{i j}
$$

The last term represents the decoherence and deexcitation of the system due to spontaneous emission and is given by

$$
[\Gamma \rho]_{i j}=\rho_{i j} \sum_{k} \frac{1}{2}\left(A_{i k}+A_{k j}\right)-\delta_{i j} \sum_{k} \rho_{k k} A_{k i},
$$

where $A_{m n}$ are spontaneous emission rates from state $m$ to state $n$, and $\delta_{i j}$ is the Kronecker $\delta$. Within this convention, $A_{m n}=0$ unless $m>n$. The set of six coupled differential equations are solved numerically, using a simple Mathematica code [21]. Since the diagonal elements of the density matrix represent the relative populations of the levels, a direct comparison can be made between the solutions of Eq. (2) and the experimental results.

In this model no assumptions have been made as to the adiabaticity of the system. However, the following assumptions have been made: the rotating wave and dipole approximations in writing the Hamiltonian in the simple form of Eq. (1); the monochromaticity of the light, in spite of it being pulsed; representing the rather complicated atomic system as being composed of only three levels, and consisting of isolated, noninteracting atoms; the modeling of the optical pulses of light as being of spatially uniform intensity and Gaussian in time. The consequences of these simplifying assumptions are discussed in Sec. IV. 


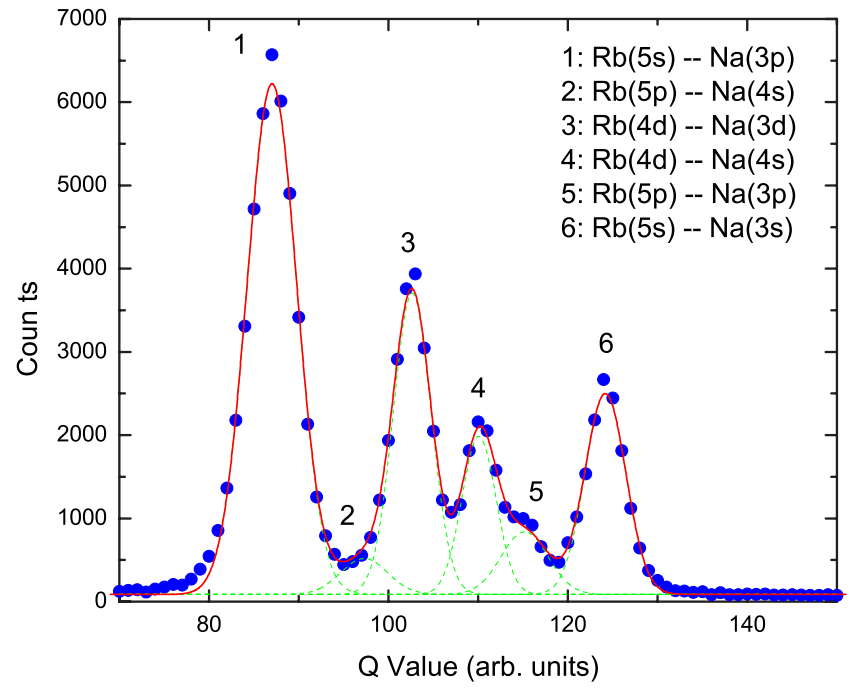

FIG. 2. (Color online) Typical $Q$-value spectrum for $7 \mathrm{keV} \mathrm{Na}^{+}$ colliding with $\mathrm{Rb}$, showing charge capture from all of the levels of interest.

\section{EXPERIMENT}

The MOTRIMS apparatus and methodology have been described in detail elsewhere [22,23]. The use of MOTRIMS as a diagnostic of the excited fraction of atoms in a MOT has also been described [24-26]. Therefore, only a brief description of the technique will be given here. Recoil ion momentum spectroscopy (RIMS) [27] consists of measuring the momentum "kick" given to a target "recoil" ion after undergoing charge transfer in collision with a projectile ion. The component of the momentum that lies along the collision axis, given by the time of flight (TOF) in this apparatus, is related to the so-called $Q$ value of the collision by

$$
Q=-\frac{m_{e} v_{P i}^{2}}{2}-v_{P i} p_{R}
$$

where

$$
Q \equiv E_{\text {initial }}^{\text {binding }}-E_{\text {final }}^{\text {binding }} .
$$

Here, $m_{e}$ is the mass of the electron, $v_{P i}$ is the projectile velocity, $p_{R \|}$ is the component of the recoil ion momentum that is parallel to the collision axis, and $E^{\text {binding }}$ refers to the binding energy of the transferred electron.

For a $Q$-value measurement to be meaningful, the thermal momentum distribution of the target must be small compared to the momentum kick given during the collision. Therefore, RIMS requires that the target be translationally cold. When the target atoms are cooled and trapped in a magneto-optical trap this variation of RIMS is known as MOTRIMS [28-30]. A sample $Q$-value spectrum for $7 \mathrm{keV} \mathrm{Na}+\mathrm{Rb}$ is shown in Fig. 2.

The area under a peak in the $Q$-value spectrum is proportional to the product of the cross section for that collision channel and the population of the initial state of the target atom. Because the relative capture cross sections for all of the channels shown in Fig. 2 have been measured $[23,24,31,32]$, the relative populations of the $\mathrm{Rb}$ atoms can be determined by dividing the areas of the peaks in Fig. 2 by the relative cross sections. Because the ion beam continuously interacts with the Rb atoms, $Q$-value spectra can be taken as a function of time, and the temporal evolution of the $\mathrm{Rb}$ populations can be measured [24-26].

The recoil momentum spectrometer, described in detail in $[22,23]$, consists of a weak electric field which directs the ionized $\mathrm{Rb}$ atoms to a field-free drift region, and then onto a two-dimension position-sensitive detector (PSD). The flight time of the recoiling $\mathrm{Rb}^{+}$, with some minor correction from the position information, gives a measurement of $p_{\|}$, and therefore, $Q$ in Eq. (4). This flight time is measured by starting a time-to-digital converter (TDC) with a signal derived from the detection of the neutralized Na projectile, and stopping the TDC with a signal derived from the $\mathrm{Rb}^{+}$recoil ion. The temporal resolution of the system is limited by the slight spread in the projectile energy, about $1 \mathrm{eV}$ out of $7 \mathrm{keV}$. In normal MOT operation, when the trapping laser is always on, the total population is split between the ground state and the first excited state. However, for the coherent excitation process under study here, we want to start with all the population in the ground state. The trapping laser is therefore turned off for $500 \mathrm{~ns}$, a time that is long compared to the lifetime of $\mathrm{Rb}(5 p)$, about $27 \mathrm{~ns}$, but short compared to the time required for atoms to escape from the trapping region, a few milliseconds [25]. After the STIRAP excitation sequence, described below, the trapping laser is turned back on, allowing the atoms to replenish and come back to positional and thermal equilibrium. A complete trapping-excitation cycle lasts $5 \mu \mathrm{s}$, and is repeated throughout the duration of the experiment.

The MOTRIMS apparatus consists of a fairly standard MOT [33] composed of a trapping and repump laser, and anti-Helmholtz coils, combined with a $\mathrm{Na}^{+}$ion gun, collision chamber, and a recoil momentum spectrometer. Details on controlling all of the lasers used in this experiment are described in the Appendix. Briefly, the trapping laser operates on the $5 s_{1 / 2},(F=2) \rightarrow 5 p_{3 / 2},(F=3)$ cycling transition, with the repump laser operating on the $5 s_{1 / 2},(F=1) \rightarrow 5 p_{3 / 2}$, $(F=2)$ transition. As described in Sec. II, coherent excitation of the $\mathrm{Rb}$ requires the presence of two additional optical pulses, the pump and Stokes lasers, pulsed at the appropriate times. For maximum population transfer efficiency, these are kept in two-photon resonance. The pump and the Stokes pulses are injected into optical fibers in order to minimize the temporal shearing of the beam that can be caused by an acousto-optical modulator (AOM), then combined using a long-pass filter before being directed into the trapping chamber. These pulses are turned on approximately $200 \mathrm{~ns}$ after the trap laser has been turned off, giving adequate time for the atoms excited to the $5 p_{3 / 2}$ state during the trapping process to relax. Because the trap laser is off for a total of $500 \mathrm{~ns}$, this leaves ample time to observe the decay of the $4 d_{5 / 2}$ atoms excited during the STIRAP process. Figure 1(b) shows sample pump and Stokes pulses measured with photodiodes. Due to an impedance mismatch, ringing occurs in the PIN diode used to measure the pump pulse, giving rise to the spurious secondary peak near $100 \mathrm{~ns}$. One can also see from Fig. 1(b) that the optical pulses are not actually Gaussian as approximated in the theoretical model, but are some- 
what flat-topped and have rather sharp cutoffs. The hyperfine levels in the uppermost level of the ladder system are unresolved. The widths of the two pulses, while near $50 \mathrm{ns,} \mathrm{are}$ not identical, with the pump pulse being a bit more narrow than the Stokes pulse. In our experiment, $\Delta_{1} /(2 \pi)=$ $-53 \mathrm{MHz}$ and $\Delta_{2} /(2 \pi)=+53 \mathrm{MHz}$, so that the excitation is under the two-photon resonance condition. The peak intensities of the pump and Stokes laser beams at the location of the MOT are determined using a power meter and a scanning knife-edge beam profiler [34] to be $1.9 \mathrm{~W} / \mathrm{cm}^{2}$ and $0.6 \mathrm{~W} / \mathrm{cm}^{2}$, respectively, giving peak Rabi frequencies of approximately 0.9 and 0.8 angular $\mathrm{GHz}$, respectively.

In the experiments described here, we wished to measure the time evolution of the populations in a three-level ladder system for different delays between the pump and Stokes pulses. To do this, a piece of electronic circuitry was constructed [35] in which an input targeted third level (TTL) pulse is delayed by $n \times 10 \mathrm{~ns}$, where $n$ is an integer between 0 and 15 , and is incremented by the circuit at regular intervals. Thus, the TTL pulse is delayed by times of 0 to $150 \mathrm{~ns}$ in steps of $10 \mathrm{~ns}$, each step lasting approximately $5 \mathrm{~s}$. The input TTL pulse had a fixed time delay with respect to the firing of the AOM that controls the Stokes laser pulse, while the delayed TTL pulse had a fixed time delay with respect to the firing of the pump laser pulse. This caused the Stokes laser pulse to be stepped in time with respect to the pump laser pulse. The fixed delays were adjusted such that for $n$ $=7$, the pump and Stokes pulses arrived at the MOT at the same time. That is, the Stokes pulse precedes the pump pulse by up to $70 \mathrm{~ns}$ (counterintuitive order) and advances on the pump pulse in $10 \mathrm{~ns}$ increments until it trails the pump pulse by up to $80 \mathrm{~ns}$ (intuitive order). We refer to the delay between the pump and Stokes pulses as $\tau$, a negative value of $\tau$ means that the Stokes pulse precedes the pump pulse (counterintuitive order), while a positive value of $\tau$ means that the Stokes pulse trails the pump pulse (intuitive order). The cycling of the pump (Stokes) delays was done asynchronously with respect to the trap (excitation) cycle, and continued throughout the data acquisition time. A signal from the delay circuit was sent as a stop to the same TDC that was started with a neutral $\mathrm{Na}$ projectile; this allows charge transfer events to be correlated with the appropriate value of $\tau$ for each real charge transfer event.

A third stop signal, produced from the delay circuit with a fixed delay from the pump pulse, was also sent to the TDC. In this manner the $Q$-value spectra were taken as a function of time, giving a measurement of the temporal evolution of the system necessary for studying the population dynamics during coherent excitation. A typical contour plot of chargetransfer counts versus $Q$ value and this TDC signal, for a single value of $\tau$ is shown in Fig. 3 .

In this figure, the right-most vertical stripe corresponds to the $\mathrm{Rb}(5 s) \rightarrow \mathrm{Na}(3 s)$ channel; the neighboring broken stripe corresponds to the $\mathrm{Rb}(5 p) \rightarrow \mathrm{Na}(3 p)$ channel (with the gap due to the MOT laser light being shut off); and the bright spot that appears inside the gap corresponds to the $\mathrm{Rb}(4 d)$ $\rightarrow \mathrm{Na}(3 d)$ channel. When the number of counts in each stripe is divided by the appropriate relative cross section, this plot carries all the necessary information about the population of

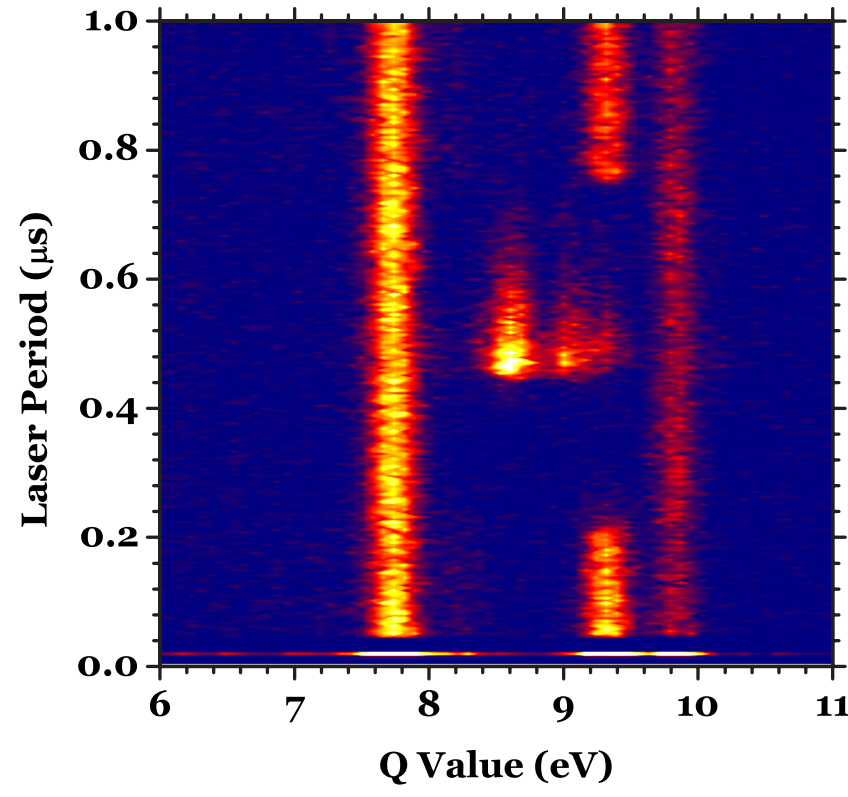

FIG. 3. (Color online) Evolution of the $Q$-value spectrum with time. The vertical stripes in this figure correspond to the peaks in Fig. 2. The thin horizontal stripe at the bottom of the plot is an artifact of the TDC.

the $\mathrm{Rb}$ atoms in the MOT: A projection along the vertical axis yields a $Q$-value spectrum like the one shown in Fig. 2, while the horizontal projection of a given stripe gives the time evolution of the corresponding state. For this work we choose the stripes corresponding to the peaks labeled 6, 5, and 3 in the $Q$-value spectrum of Fig. 2 in order to obtain, respectively, the populations of $\mathrm{Rb}(5 s), \mathrm{Rb}(5 p)$, and $\mathrm{Rb}(4 d)$. One can see from the $Q$-value plot of Fig. 2 that for large $\mathrm{Rb}(4 d)$ populations the peak labeled 5 is convoluted with the peak labeled 4. It would seem that this would make the determination of the $\mathrm{Rb}(5 p)$ population problematic. However, this turns out not to be a difficulty. Both peaks 3 and 4 correspond to capture from $\mathrm{Rb}(4 d)$. Therefore, no matter what the excitation mechanism, the ratio of the areas of peak 3 to peak 4 is a constant; we call it $R$. Under conditions where the $\operatorname{Rb}(5 p)$ population was extremely small, this ratio was measured. Thus, the area under the peak labeled 5 was determined by measuring the total area under peaks 5 and 4 , and subtracting from this the area of peak 3 divided by $R$. That is,

$$
A_{5}=A_{5+4}-A_{3} / R \quad \text { with } R \equiv A_{3} / A_{4} .
$$

Here, $A$ refers to peak areas and the subscripts refer to the peak labels of Fig. 2. In this way, we could quickly and accurately determine the relative populations of all three levels without the use of curve fitting procedures, and their associated uncertainties.

\section{RESULTS}

Figure 4 shows the fractional populations of the $\mathrm{Rb}(5 s)$, $\mathrm{Rb}(5 p)$, and $\mathrm{Rb}(4 d)$ states as functions of time, for five representative values of $\tau$. The data are shown as points, while 


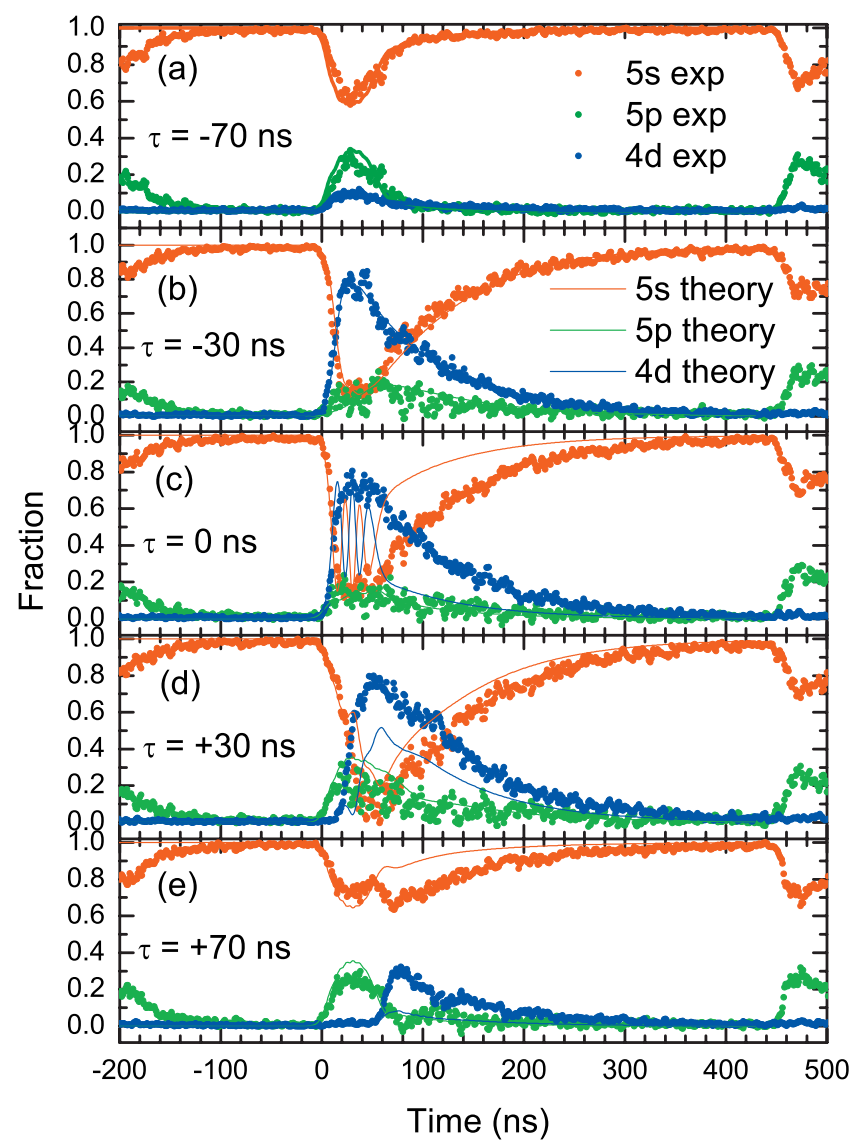

FIG. 4. (Color) The time evolution of the relative $5 s, 5 p$, and $4 d$ populations for different temporal delays between the pump and the Stokes laser. Out of the 16 different cases which were investigated, only five are shown, two for the counterintuitive order (-70 ns and $-30 \mathrm{~ns}$, respectively), the overlap case, and two more for the intuitive order (30 ns and $70 \mathrm{~ns}$, respectively).

calculations from the simple model of Sec. II are shown as solid lines. In all cases shown in Fig. 4 the calculations were done with no fitted parameters. For both theory and experiment, the $\mathrm{Rb}(5 s)$ population is shown in red, the $\mathrm{Rb}(5 p)$ population is in green, and the $\mathrm{Rb}(4 d)$ population is in blue. The scatter in the data points is the best indication of relative error; however, to estimate absolute error in population fractions, more detailed analysis was required.

There are several sources of uncertainty that contribute to the overall error in measured absolute population fractions. The first of these is the uncertainty in relative charge transfer cross sections, $\sigma_{5 p-3 p} / \sigma_{5 s-3 s}, \quad \sigma_{4 d-3 d} / \sigma_{5 s-3 s}, \quad$ and $R$ $\equiv \sigma_{4 d-3 d} / \sigma_{4 d-4 s}$, where the $\sigma s$ are the cross sections for the transfer channels indicated with subscripts. As previously discussed $[23,26,31,32,36]$, the error in the cross section ratios is dominated by counting statistics and background subtraction. The second potential source of error is the assumption that the ion beam is sampling the same volume of MOT target that the combination of pump and Stokes lasers are exciting. In this experiment the procedure was to adjust the MOT target's position with respect to the ion beam until a maximum charge transfer count rate was obtained. The alignment of the Stokes and pump lasers was then adjusted until a maximum in molecular ion signal, $\mathrm{Rb}_{2}^{+}$, formed through photoassociative ionization $[37,38]$ was obtained [39]. It was found that the alignment of these two lasers was not terribly critical, indicating that their spot size was comparable to the size of the MOT cloud. The ion beam diameter is greater than the laser spot sizes and we therefore conclude that the ion beam is sampling the entire MOT, and that the entire MOT is exposed to the pump and Stokes beams. The overall uncertainty in population fraction is then dominated by the cross section error and is estimated to be $2.5 \%, 1.6 \%$, and $2.4 \%$ for $\operatorname{Rb}(5 s), \operatorname{Rb}(5 p)$, and $\mathrm{Rb}(4 d)$, respectively.

In Fig. 4(a), the temporal delay between the pump and the Stokes laser pulse is $-70 \mathrm{~ns}$. This leads to little population transfer since the two laser pulses are barely overlapping in time. The $a b$ initio calculation fits the data extremely well. When the Stokes laser precedes the pump laser by $30 \mathrm{~ns}$, as shown in Fig. 4(b), a maximum of nearly $90 \%$ of the population is transferred to the $\mathrm{Rb}(4 d)$ state. The agreement with the calculated temporal evolution is again extremely good. Note how the population of the $\operatorname{Rb}(5 p)$ state lags that of the $\mathrm{Rb}(4 d)$; this is because the $\mathrm{Rb}(5 p)$ state is populated nearly entirely by spontaneous emission from the $\mathrm{Rb}(4 d)$ state, with very little direct excitation from the $\mathrm{Rb}(5 s)$ state. This is one of the signatures of the STIRAP process. In Fig. 4(c), the two laser pulses are introduced at the same time. The calculated temporal evolution shows one- and two-photon Rabi oscillations which, however, are not seen in the experiment. We believe that this is because the atoms in the MOT are exposed to a range of intensities from the Stokes and pump lasers, whereas in the calculation, a single representative intensity is used. Thus in the experiment, the Rabi oscillations are washed out. Figure 4(d) shows the population evolution when the Stokes and pump lasers arrive in the intuitive order, with the pump laser now preceding the Stokes laser by $30 \mathrm{~ns}$. The agreement with the theory is much less satisfactory. Nevertheless there are some points of agreement: the general shapes of population peaks are in agreement, even if the amplitudes are not. Furthermore, a clear signature of sequential excitation is given by the appearance of population in the $\mathrm{Rb}(5 p)$ state before the $\mathrm{Rb}(4 d)$ state. In Fig. 4(e), the pump laser precedes the Stokes laser by $70 \mathrm{~ns}$. Again, the $\mathrm{Rb}(5 p)$ state is populated well before the $\mathrm{Rb}(4 d)$ state, as expected in a sequential excitation process. As the Stokes laser further excites the atoms to the $\mathrm{Rb}(4 d)$ state, the $\mathrm{Rb}(5 p)$ population drops, but is somewhat revived by spontaneous emission from the $\mathrm{Rb}(4 d)$ state. The agreement with theory is not very good since neither the revival in the $\mathrm{Rb}(5 p)$ population nor the resulting revival in the $\mathrm{Rb}(4 d)$ population, both of which are clear in the experiment (and make sense intuitively) appear in the theory. To summarize Fig. 4, the temporal evolution of the $\mathrm{Rb}(5 s), \mathrm{Rb}(5 p)$, and $\mathrm{Rb}(4 d)$ populations are clear and consistent with what one might qualitatively expect. However, only in the so-called counterintuitive cases does the simple theoretical model give good agreement with experiment.

Figure 4 is fundamentally different from all other STIRAP results to date: It shows the temporal development of all the levels of interest. It is especially important to have measurements of this type in order to more fully test theo- 
retical models. For example in Figs. 4(c)-4(e), there exist disagreement between theory and experiment. However, if the populations were integrated over time-and even worse, if only the population of the $\mathrm{Rb}(4 d)$ level were shown - then the disagreement would not appear so great, nor would there be any hints as to the sources of the disagreement.

However, comparison with theory is not the only reason that seeing the temporal evolution is important. Different applications of STIRAP can have different requirements. For example, in the potential HHG application [15] mentioned in the introduction, it would be important to have the ultrafast laser pulse arrive in the $\mathrm{Rb}$ vapor while the $\mathrm{Rb}(4 d)$ state is maximally populated, without regard to the $\mathrm{Rb}(5 p)$ population. As another example, suppose one has a three-level system and one is trying to derive a particular chemical reaction which is favored if the system is in the targeted "third" level, regardless of which states the remaining atoms or molecules are in. Then what may matter the most, depending on how the reaction scales with population, is either the peak or the integrated population, integrated if the reaction rate is linear in population; peak if the reaction rate is greater than linear. Furthermore, even if the reaction rate is only linear in population, the temporal evolution of that population would help one predict the temporal evolution of the reaction rate. As a final example, suppose the desired chemical reaction is with the "third" level, but an undesirable reaction takes place with atoms or molecules in the intermediate level. Then, the figure of merit for excitation may be the instantaneous ratio between the "third" and intermediate levels. This can only be estimated from a knowledge of the temporal evolution of all three levels.

It was noted earlier that the agreement between theory and experiment was extremely good for the counterintuitive ordering of the pulses, but less satisfactory for overlapping pulses and pulses that arrived in the intuitive order. We believe that the discrepancy is due to a break down in the model's approximations. The first of these were the rotating wave and dipole approximations. We believe that for the intensities, optical frequencies, and pulse lengths used in the experiment, these should be completely valid. The second approximation was that the optical pulses were monochromatic. This was not strictly correct in that the $\mathrm{cw}$ bandwidth of our lasers was roughly $1 \mathrm{MHz}$, and going from $\mathrm{cw}$ to pulsed broadens the bandwidth to about $10 \mathrm{MHz}$. However, this linewidth is small compared to the energy splitting of the relevant levels. It was further assumed that the rather complicated atomic system under study could be approximated by a three-level system. This assumption is probably less valid because it neglects the hyperfine splitting in the $\mathrm{Rb}\left(4 d_{5 / 2}\right)$ and $\mathrm{Rb}\left(5 p_{3 / 2}\right)$ states. Furthermore, due to the magnetic field gradient in the MOT, there is a spatially dependent splitting between the $m_{F}$ levels. The effect of these additional levels is unknown; the solution is to take into account the hyperfine structure, and perhaps even try to include a sum over Zeeman-split magnetic sublevels to see how the predictions of the calculations change. The target is an optically thick cloud of many atoms; the MOT is running at the radiation trapping limit. Moreover, we have observed photoassociation [39] in this system. Thus, the assumption of an optically thin, noninteracting atomic target is suspect. We do not know if or how this could impact the validity of the theoretical model. When the calculations were made, single fixed values were used for intensities of the pump and Stokes lasers. These corresponded to the measured peak values for the corresponding optical pulses. However, the beams from both the pump and Stokes lasers were focused to be comparable in size to the MOT, which means that the intensities were different in different parts of the MOT, whereas the ion beam was sampling the entire MOT. Thus, while the measured populations should be accurate, the calculated fractions, which were based on the peak intensities, could be in error. Furthermore, because the measurement spatially averaged over the laser intensities, it is not at all surprising that the Rabi structure of the theoretical model at $\tau=0$ was not seen experimentally. In the calculations, it was assumed that the temporal profiles of the optical pulses were Gaussian. However, as seen in Fig. 1(b), the actual profiles were poor imitations of Gaussians. With this in mind, the temporal match of the theory to experiment is surprisingly good.

\section{SUMMARY}

In this paper we described the measurement of the temporal evolution of all relevant states in a three-level ladder system undergoing coherent excitation through the STIRAP process. The measurement was done using charge transfer as a probe having a temporal resolution of a few nanoseconds. These STIRAP measurements show the temporal evolution of all the relevant levels. Furthermore, the measurements were absolute in the sense that no normalization to theory was needed to put the relative populations on an absolute scale. The experimental results were compared with the predictions from a simple model of coherent excitation. In the "counterintuitive" ordering of the pulses, the simple model was in excellent agreement with experiment. However, in the "intuitive" ordering cases, the agreement was significantly less satisfactory. Possible reasons for this were discussed.

Nevertheless, the experiment demonstrated some important points. For example, we see that even the simple model seems robust enough to work pretty well when the pulses are in the counterintuitive order. Moreover, the experimental results can be used to test more sophisticated models in which more levels and a spatially varying intensity can be included.

In future work we will refine the model in the manner just mentioned. In addition, we plan to change other excitation parameters, including the one- and two-photon detunings from resonance, and the intensities of the Stokes and pump lasers. These results will be compared to predictions that have already been presented [19]. An additional area to explore concerns adiabaticity. In the experiment done here, the period associated with the Rabi frequency was short compared to the time scale over which the laser intensities were varied. Loosely speaking, this is the condition for adiabatic excitation and is critical for STIRAP. In future experiments we will deviate from this adiabatic condition to see if the theoretical predictions are correct. There has been a great deal of interest recently in using Rydberg atoms for quantum information applications $[7,40-42]$. For this to be feasible, a necessary condition is that the atoms can be efficiently ex- 


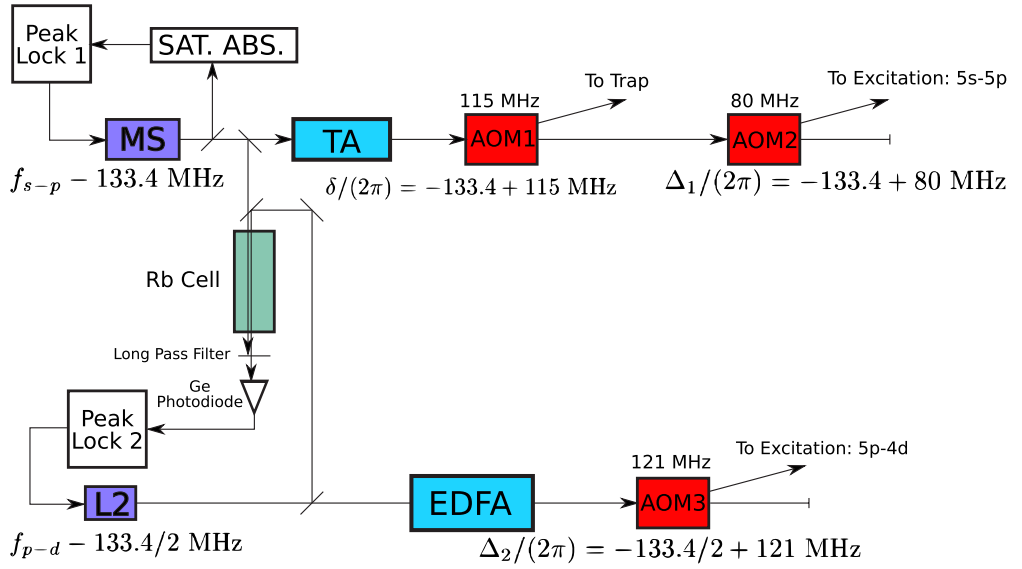

FIG. 5. (Color online) Locking scheme for the trapping, pump, and Stokes lasers. Details are provided in the text.

cited. One possible scheme is to excite the Rydbergs along the four-level ladder sequence $5 s-5 p-4 d-n f$. According to theory [43] when even numbers of levels are involved, STIRAP behaves in a fundamentally different manner than for an odd number of levels. We therefore plan to study this four-level ladder system using an approach similar to that used in this work, and compare the measured populations of all the levels with the predictions of theory.

\section{ACKNOWLEDGMENTS}

The authors would like to express their appreciation to Professor K. Corwin for lending the erbium-doped fiber amplifier that was essential for obtaining the required optical power for the Stokes laser. This work was supported by the Chemical Sciences, Geosciences, and Biosciences Division, Office of Basic Energy Sciences, Office of Science, U. S. Department of Energy.

\section{APPENDIX: LOCKING THE LASERS}

Although there is nothing special in the laser control techniques implemented here, details are presented because precise control of all lasers was critical to the success of this experiment. The optical system is shown schematically in Fig. 5. An external cavity diode laser, labeled MS, is locked to the 2-3 crossover transition in ${ }^{87} \mathrm{Rb}$, which lies 133.4 MHz below the $5 s_{1 / 2},(F=2) \rightarrow 5 p_{3 / 2},(F=3)$ transition in a saturated absorption spectrum. This "master laser" is locked using a Zeeman dithering scheme [44] labeled "Peak Lock1" in Fig. 5. Most of the power of the master laser is sent to a tapered amplifier (TA), the output of which is directed through an acousto-optical modulator, labeled AOM1 in Fig. 5. This AOM is then used to shift the laser light by $115 \mathrm{MHz}$ to the blue, bringing it to $\delta / 2 \pi=18 \mathrm{MHz}$ on the red side of the $5 s_{1 / 2},(F=2) \rightarrow 5 p_{3 / 2},(F=3)$ resonance. This frequency-shifted output from AOM1 is used to trap and cool ${ }^{87} \mathrm{Rb}$. AOM1 also serves as a convenient on-off switch for the trapping light; when the trap is off, all of the power from the TA passes, unshifted, through AOM1, and is injected into a second AOM, referred to in Fig. 5 as AOM2. This is used both to create the pump pulse and to control its detuning from single photon resonance with the $5 s_{1 / 2},(F=2) \rightarrow 5 p_{3 / 2}$, $(F=3)$ transition. In the experiment described here, AOM2 is held at a fixed frequency of $80 \mathrm{MHz}$, making the detuning equal to $53 \mathrm{MHz}$. The zero-order output of AOM2 is directed to a beam dump.

A fraction of the unamplified master laser is directed through a Rb vapor cell, where it excites the particular Doppler group of atoms that are blueshifted into resonance. A fraction of the $1529 \mathrm{~nm}$ light from a second external cavity diode laser, referred to as "L2," is overlapped with light from the master laser and passes through the $\mathrm{Rb}$ cell colinearly with it. As the frequency of L2 is dithered, absorbtion by the $5 p_{5 / 2}$ levels in $\mathrm{Rb}$ can be clearly seen and are used to lock L2. The light from L2 only interacts with the same Doppler group that was excited by the master laser. Therefore, L2 is locked $133.4 / 2 \mathrm{MHz}$ to the red of the $5 p_{3 / 2},(F=3) \rightarrow 5 p_{5 / 2}$, $(F \sim 4)$ transitions. (The factor of 2 arises because the frequency of the light from L2 is one-half of that from the master laser.) Most of the light from L2 is amplified using an erbium-doped fiber amplifier, before being injected into a third AOM, referred to as AOM3 in Fig. 5. AOM3 is operated at $121 \mathrm{MHz}$, shifting the light from L2 $53 \mathrm{MHz}$ to the blue of the $5 p_{3 / 2},(F=3) \rightarrow 5 p_{5 / 2},(F \sim 4)$ transition, and thus bringing the pump and Stokes lasers into two-photon resonance.

The repump laser, not shown in Fig. 5, is tuned to the $5 s_{1 / 2},(F=1) \rightarrow 5 p_{3 / 2},(F=2)$ transition in a second saturated absorption setup, again using a Zeeman dithering scheme. Light from this laser is left on at all times; thus no AOM is required for this laser. 
[1] B. W. Shore, The Theory of Coherent Atomic Excitation (Wiley, New York, 1990), Vol. 2.

[2] B. W. Shore, The Theory of Coherent Atomic Excitation (Wiley, New York, 1990), Vol. 1.

[3] N. V. Vitanov, M. Fleischhauer, B. W. Shore, and K. Bergmann, Adv. At., Mol., Opt. Phys. 46, 55 (2001).

[4] F. Vewinger, M. Heinz, R. G. GarciaFernandez, N. V. Vitanov, and K. Bergmann, Phys. Rev. Lett. 91, 213001 (2003).

[5] T. Peters, L. P. Yatsenko, and T. Halfmann, Phys. Rev. Lett. 95, 103601 (2005).

[6] T. Cubel, B. K. Teo, V. S. Malinovsky, J. R. Guest, A. Reinhard, B. Knuffman, P. R. Berman, and G. Raithel, Phys. Rev. A 72, 023405 (2005).

[7] M. D. Lukin, M. Fleischhauer, R. Cote, L. M. Duan, D. Jaksch, J. I. Cirac, and P. Zoller, Phys. Rev. Lett. 87, 037901 (2001).

[8] J. Sørensen, D. Møller, T. Iversen, J. Thomsen, F. Jensen, P. Straanum, D. Voigt, and M. Drewsen, New J. Phys. 8, 261 (2006).

[9] P. Marte, P. Zoller, and J. L. Hall, Phys. Rev. A 44, R4118 (1991).

[10] U. Gaubatz, P. Rudecki, S. Schiemann, and K. Bergmann, J. Chem. Phys. 92, 5363 (1990).

[11] K. Winkler, F. Lang, G. Thalhammer, P. v. d. Straten, R. Grimm, and J. H. Denschlag, Phys. Rev. Lett. 98, 043201 (2007).

[12] H. Y. Ling, H. Pu, and B. Seaman, Phys. Rev. Lett. 93, 250403 (2004).

[13] U. Hohenester, F. Troiani, M. Molinari, G. Panzarini, and C. Macchiavello, Appl. Phys. Lett. 77, 1864 (2000).

[14] T. G. Walker (private communication).

[15] P. M. Paul, T. O. Clatterbuck, C. Lyngå, P. Colosimo, L. F. DiMauro, P. Agostini, and K. C. Kulander, Phys. Rev. Lett. 94, 113906 (2005).

[16] K. Bergmann, H. Theuer, and B. W. Shore, Rev. Mod. Phys. 70, 1003 (1998).

[17] W. Süptitz, B. C. Duncan, and P. L. Gould, J. Opt. Soc. Am. B 14, 1001 (1997).

[18] H. A. Camp, Ph.D. thesis, Kansas State University, 2005.

[19] H. A. Camp, M. H. Shah, M. L. Trachy, O. L. Weaver, and B. D. DePaola, Phys. Rev. A 71, 053401 (2005).

[20] Our laser does not resolve the hyperfine states $F=1-4$ in ${ }^{87} \mathrm{Rb}\left(4 d_{5 / 2}\right)$.

[21] Available upon request from the auhtors of this paper.

[22] H. Nguyen, X. Fléchard, R. Brédy, H. A. Camp, and B. D. DePaola, Rev. Sci. Instrum. 75, 2638 (2004).
[23] H. Nguyen, Ph.D. thesis, Kansas State University, 2003.

[24] X. Fléchard, H. Nguyen, R. Brédy, S. R. Lundeen, M. Stauffer, H. A. Camp, C. W. Fehrenbach, and B. D. DePaola, Phys. Rev. Lett. 91, 243005 (2003).

[25] R. Brédy, H. Nguyen, H. A. Camp, X. Fléchard, and B. D. DePaola, Nucl. Instrum. Methods Phys. Res. B 205, 191 (2003).

[26] M. H. Shah, H. A. Camp, M. L. Trachy, G. Veshapidze, M. A. Gearba, and B. D. DePaola, Phys. Rev. A 75, 053418 (2007).

[27] V. Mergel et al., Phys. Rev. Lett. 74, 2200 (1995).

[28] M. van der Poel, C. V. Nielsen, M. A. Gearba, and N. Andersen, Phys. Rev. Lett. 87, 123201 (2001).

[29] J. W. Turkstra, R. Hoekstra, S. Knoop, D. Meyer, R. Morgenstern, and R. E. Olson, Phys. Rev. Lett. 87, 123202 (2001).

[30] X. Fléchard, H. Nguyen, E. Wells, I. Ben-Itzhak, and B. D. DePaola, Phys. Rev. Lett. 87, 123203 (2001).

[31] M. H. Shah, H. A. Camp, M. L. Trachy, X. Fléchard, M. A. Gearba, H. Nguyen, R. Brédy, S. R. Lundeen, and B. D. DePaola, Phys. Rev. A 72, 024701 (2005).

[32] T. G. Lee, H. Nguyen, X. Fléchard, B. D. DePaola, and C. D. Lin, Phys. Rev. A 66, 042701 (2002).

[33] E. L. Raab, M. G. Prentiss, A. Cable, S. Chu, and D. E. Pritchard, Phys. Rev. Lett. 59, 2631 (1987).

[34] G. Veshapidze, M. L. Trachy, M. H. Shah, and B. D. DePaola, Appl. Opt. 45, 8197 (2006).

[35] H. U. Jang, J. Blieck, G. Veshapidze, M. L. Trachy, and B. D. DePaola, Rev. Sci. Instrum. (to be published).

[36] H. Nguyen, R. Brédy, H. A. Camp, T. Awata, and B. D. DePaola, Phys. Rev. A 70, 032704 (2004).

[37] K. M. Jones, E. Tiesinga, P. D. Lett, and P. S. Julienne, Rev. Mod. Phys. 78, 483 (2006).

[38] J. Weiner, V. S. Bagnato, S. Zilio, and P. S. Julienne, Rev. Mod. Phys. 71, 1 (1999).

[39] M. L. Trachy, G. Veshapidze, M. H. Shah, H. U. Jang, and B. D. DePaola (unpublished).

[40] J. M. Raimond, M. Brune, and S. Haroche, Rev. Mod. Phys. 73, 565 (2001).

[41] D. Jaksch, J. I. Cirac, P. Zoller, S. L. Rolston, R. Côté, and M. D. Lukin, Phys. Rev. Lett. 85, 2208 (2000).

[42] A. S. Sørensen, C. H. van der Wal, L. I. Childress, and M. D. Lukin, Phys. Rev. Lett. 92, 063601 (2004).

[43] J. Oreg, K. Bergmann, B. W. Shore, and S. Rosenwaks, Phys. Rev. A 45, 4888 (1992).

[44] T. P. Dinneen, C. D. Wallace, and P. L. Gould, Opt. Commun. 92, 277 (1992). 